\title{
Multiple target localization in WSNs using compressed sensing reconstruction based on $A B C$ algorithm
}

\author{
Hao Feng ${ }^{1,2}$, Junyan Chen $^{2}$ and Lei Luo ${ }^{1}$ \\ ${ }^{1}$ School of Computer Science and Engineering, University of Electronic Science and Technology of China, Chengdu 611731; \\ ${ }^{2}$ Guangxi Colleges and Universities Key Laboratory of cloud computing and complex systems, Guilin University of Electronic Technology, \\ Guilin 541004, China
}

\begin{abstract}
In allusion to the application of compressed sensing theory in multi-target localization in wireless sensor networks, the paper proposes a method of sparse signal reconstruction based on artificial bee colony algorithm and designs partial update strategy of location coding. A mode of disturbances mutation operation for food source exploitation is designed to improve the search efficiency in high-dimensional solution space; it also improves the global search efficiency of the algorithm. The comparing of simulation analysis with other algorithms verifies that the algorithm effectively solves problem of multi-target localization in wireless sensor networks and has better resistance to interference.
\end{abstract}

\section{Introduction}

Localization service as one of the important basic functions of the wireless sensor network [1], whether for the location information of the sensor node itself or for the targeting localization of the monitored area, is the basis of other applications such as environmental detection, target tracking, and structural health monitoring [2]. It can be said that the wireless sensor network will not have any effect without the location information [3]. Thus localization has been a challenging hot area on wireless sensor network. Common localization algorithms are those based on RSS (Received Signal Strength) and its improved algorithm [4], ToA [5], TDOA [6], AOA [7], and so on. Comparing with other algorithms the RSS-based algorithms has advantages as no need for special hardware, low cost, and easy access thus it has been widely studied and applied. In these localization algorithms, because of the limits of computing ability and energy consumption in sensor node, centralized algorithm has higher accuracy than distributed one. However, like other monitoring applications, in a centralized algorithm, each sensor node responsible for perception of location information needs to send the data of signal strength of each target to the sink, then the sink node with strong computing power runs centralized algorithms to recover the location of the target. In the application of multi-target localization, with a lot of wireless communication expenses, this results in rapid consumption of onboard energy in sensor node and decline in the performance of the communication network.
Compressed sensing is a new theory of data compression and reconstruction in recent years [8], the low requirements for sampling of compressed sensing system coincides with the characteristics of constrained resource in sensor nodes, resulting in its quick application in data collection, topology-aware and other aspects of wireless sensor networks. Meanwhile compressed sensing also offers new ideas and methods for the study of localization issues of wireless sensor networks. Cevher et al.[9] studied WSNs localization issues with CS, but this method requires a localization dictionary of sensor nodes, which increases storage expense of the overhead int the sensor node, thus it's unacceptable for the low-cost sensors with small memory. A grid-based WSNs localization problem is modeled as $\mathrm{N}$-dimensional reconfiguration when $\mathrm{K}$ sparsity vectors are 1 [10]; compressed sensing is used to achieve multi targeting in a wireless sensor network; the shortcomings of this approach is that WSN data can't be further compressed, which is, to $\mathrm{M}$ sensor nodes under the situation of $\mathrm{K}$ targets, $\mathrm{M} \times \mathrm{K}$ perception data need to be transferred. In recent years, among some works [11] [12] [13] [14], target location has been studied via the model and solving method with compressed sensing respectively, and has made some progress.

This paper presents a reconstruction method of compression perception based on discrete artificial bees colony algorithm to localize multi-target in wireless sensor network. This method transfers the location information of target into a discrete encoding of food source in artificial honey bees system, obtaining suboptimal information of location reconstruction by foraging bees iterate operation, therefore achieves signal 
reconstruction with the unknown sparsity, consequently by constructing an effective measurement matrix and sparse matrix, the WSNs targeting problem is transformed into reconstruction problem of compressed sensing signals, which enables multi-target localization in WSNs. Finally, it shows better performance by verifying the effectiveness of the proposed method through simulation analysis and comparing with other recovery algorithms.

\section{Problem Description and System Model}

Assuming the square monitored region is divided into $N$ grids, in this monitored region, we deploy randomly $M$ position-known sensors. There are $K$ ( $K$ is unknown) targets to be located in the entire monitored area. Sensor nodes are used to measure the signals of energy of the targets within the monitored region, finally to recover the position information of the targets based on grids in the fusion center.

The system works as follows: each target periodically transmits signal by broadcasting, the transmission cycle is $T$, and each target is independent without synchronization requirement. The sensor node periodically collects signals, the collection cycle is $T$ as well, the node simply adds up the strength values of the received signals within the cycle. After the end of the time slice of the cycle, each sensor node sends the results collected to the fusion center, then the center runs signal reconstruction algorithm and gets the ultimate locations of targets in the grid.

Assuming the energy value emitted by $K$ signals can be linear superposition, then the energy $e_{i}$ received by $i$-th sensor is:

$$
e_{i}=\sum_{j=1}^{K}\left(P_{i, j}+N_{i}\right), P_{i, j}=P_{0}-10 n_{p} \lg \left(D_{i, j} / D_{0}\right)
$$

$N_{i} \sim N\left(0, \delta_{i}^{2}\right)$ is the Gaussian white noise; $P_{i, j}$ is signal strength received of the $k$-th target by $i$-th sensor, the measure unit is $\mathrm{dB} ; P_{0}$ represents the received signal strength when the reference distance is $D_{0} ; n_{p}$ is the path attenuation exponent with typically values between 2 and 4; $D_{i, j}$ is the Euclidean distance between the target locations and sensor $i$.

For $N$ grids, the target position is defined as the grid center, the available vector of target location can be represented as $s=\left[s_{1}, \ldots, s_{i}, \ldots, s_{N}\right]^{T}$. When there is targets in the $i$-th grid, $s_{i}=1$; otherwise $s_{i}=0$. Obviously $s$ is the $N \times 1$ vector $(\mathrm{K}<<\mathrm{N})$ when sparsity degree is $K$. Therefore WSNs multi-targeting problem can be converted to the sparse approximation problem with compressed sensing theory and sparse signal reconstruction method for the target's sparse vector in spatial location. Interference noise increases the uncertainty of signal reconstruction and challenges the compression-aware application in multi-target localization.

\section{Compressed Sensing Localization and Measurement Matrix Construction}

The general process of compressed sensing [15] [16] is: a known measurement matrix $\boldsymbol{\Phi}$ belongs to $R_{M \times N}(M<<N)$ and the linear measurement value $Y=R_{M \times 1}$ of some unknown signal $s=R_{N \times 1}$ when using the measurement matrix as formula (2):

$$
Y_{M \times 1}=\boldsymbol{\Phi}_{M \times N} \times \boldsymbol{s}_{N \times 1}
$$

$Y$ can be seen as a linear projection of signal $s$ in the measurement matrix $\boldsymbol{\Phi}$. The main objective of compressed sensing is to reconstruct unknown signal $s$ from the measurement result $Y$. Obviously, as the dimension of $s$ is far greater than the dimension of $Y,(2)$ is an solution to the problem of underdetermined linear equations, there are infinitely solutions. This problem is an NP-Hard problem that has been proven already. However compressive sensing theory has proved that if the signal $\boldsymbol{s}$ is $K$ sparse, and $Y$ and $\boldsymbol{\Phi}$ satisfy RIP (Restricted Isometry Property) condition, signal $s$ can be accurately reconstructed from the measured values $Y$ by solving the minimization problem of $l_{l}$ norm as follow:

$$
\begin{gathered}
\boldsymbol{s}_{\text {estimate }}=\arg \min \|\boldsymbol{s}\|_{1}, \\
\text { s.t. } \quad \boldsymbol{\Phi} \boldsymbol{s}=Y
\end{gathered}
$$

(3) is a programming problem can be solved by a valid method of convex optimization, which greatly promotes the applications of compressed sensing theory. One of the key techniques of compressed sensing is the design of measurement matrix. In a grid-based localization model, assuming that the target is located in the center of the grid, the measurement matrix $\boldsymbol{\Phi}$ of each element $\varphi_{i, j}$ is defined as:

$$
\varphi_{i, j}=P_{i, j}, \quad i \in[1, M], j \in[1, N]
$$

The definition of $P_{i, j}$ is the same as (1). Therefore, the sampling process of the system can be described with formula (5).

$$
\begin{aligned}
& {\left[\begin{array}{c}
y_{1} \\
y_{2} \\
\vdots \\
y_{M}
\end{array}\right]=\left[\begin{array}{c}
P_{1,1} s_{1}+P_{1,2} s_{2}+\cdots+P_{1, N} s_{N} \\
P_{2,1} s_{1}+P_{2,1} s_{2}+\cdots+P_{2, N} s_{N} \\
\vdots \\
P_{M, 1} s_{1}+P_{M, 1} s_{2}+\cdots+P_{M, N} s_{N}
\end{array}\right]} \\
& =\left[\begin{array}{cccc}
P_{1,1} & P_{1,2} & \cdots & P_{1, N} \\
P_{2,1} & P_{2,2} & \cdots & P_{2, N} \\
\vdots & \vdots & \ddots & \vdots \\
P_{M, 1} & P_{1,2} & \cdots & P_{M, N}
\end{array}\right]\left[\begin{array}{c}
s_{1} \\
s_{2} \\
\vdots \\
s_{N}
\end{array}\right]
\end{aligned}
$$

Assuming the total number of the target is $K$, obviously, the dilution of $N$-dimensional vector $s$ is $K$. the measurement result $Y$ of the sensor is the product of measurement matrix and sparse vector $s$, the corresponding physical meaning of $y_{i}$ is sum of signal intensities of multiple targets received by the sensor $i$ in each period $T$. It can be proved that the measurement matrix $\boldsymbol{\Phi}$ satisfies the RIP condition. Because of the limited space of this paper, the proof is omitted; thus the 
multi-targeting problem in sensor network converts into a reconstruction problem of sparse signals of compressed sensing, due to the observed matrix meet the conditions of RIP, we can achieve multi-target localization function with $l_{l}$ norm optimization model by using BP, OMP, GMP and other methods. This model works better in an ideal situation; but in a real environment, there is often a variety of interference affected by the deployment environment; so formula (5) in the real world is expressed as:

$$
Y_{M \times 1}=P_{M \times N} \boldsymbol{s}_{N \times 1}+N_{M \times 1}
$$

$N_{M \times 1}$ is the Gaussian white noise superimposed on the signals received by the sensor $M$. Because of the existence of signal noise, the effect of solving $l_{l}$ norm is not ideal with larger errors and false targets, so further research is needed to obtain location information with higher quality

\section{Sparse Reconstruction Method Based on Discrete ABC Algorithm}

The algorithm of sparse signal reconstruction is one of the core contents of the compressed sensing theory, the current research is moving towards stable, low computational complexity and a smaller numbers of observations [17]. ABC algorithm [18], as a new bionic swarm intelligence optimization algorithm proposed in recent years, has won the attention of academia and engineering with its good optimization ability in smaller populations, fewer iterations, less need of parameters setting in the face of high-dimensional and multi-mode optimization problem [19]. The basic ABC algorithm is designed for continuous optimization problem. In order to apply $\mathrm{ABC}$ optimization algorithm to the sparse reconstruction of signals, the paper proposes a discrete ABC algorithm, and proposes for food source development and exploitation strategies used in discrete problem while introducing a perturbation operator to enhance the ability to escape from local optimal algorithm. Here in DABCR (Discrete Artificial Bee Colony Reconstruction) algorithm, the objective function is defined as:

$$
\min f(s)=\left\|Y-\boldsymbol{\Phi}_{\boldsymbol{s}}\right\|_{2}, \boldsymbol{s} \in \mathrm{R}^{N}
$$

$\boldsymbol{s}$ is the demanded sparse signal, it is equivalent to food source coding in the algorithm; the coding rule is: when there is a target in $i$-th grid, the corresponding $s_{i}$ is 1 , otherwise is 0 . The key to discrete $\mathrm{ABC}$ algorithm is to design the location updating strategy. Classic ABC algorithm uses formula (8) for localization updating.

$$
v_{i j}=s_{i j}+r_{i j}\left(s_{i j}-s_{k j}\right)
$$

$s_{i j}$ is the $j$-th variable dimension of the $i$-th food source, $k$ is the nectar obtained by random selection using formula (9). $r_{i j}$ is a random number between $[-1,1]$. Formula (8) is for the optimization of continuous variables; in order to meet the issue model here, a food source localization updating strategy is designed as following. The definition of variables such as $s_{i j}$ still uses that in formula (8), but the value of $s_{i j}$ can only be 0 or 1 , we redefine the operation rule of subtraction and addition as:

i. If $s_{i j}$ and $s_{k j}$ are different, then $s_{i j}-s_{k j}$ equals to 1 , otherwise the result to zero.

ii. If $s_{k j}$ equals to 1 , then $s_{i j}+s_{k j}$ to 1 ; if $s_{i j}$ and $s_{k j}$ are the same, then $s_{i j}+s_{k j}$ equals to 0 , otherwise to 1 .

iii. Considering the actual situations, $s_{i j}$ value is restricted in three cases of $-1,0$ and 1 . When the number of 1 in $s_{i}$ exceeds the threshold $T_{\max }$, the position of 1 of $T_{\max }-\left\|s_{i}\right\|_{1}$ in $s_{i}$ will be changed randomly to 0 .

$$
P_{i}=f i t_{i} / \sum_{j=1}^{S N} f i t_{j}
$$

To further enhance algorithm's ability of getting out of local optimization, an operator named Perturb $\left(s_{i}\right)$ for escaping from the local minima is designed. If the searching operation in this iteration does not improve the quality of the location of food source, then disturbance operation would be executed with probability $P_{\text {perturb }}$. Disturbance process should be controlled at a moderate level, if the disturbance on the solution is too strong, then the algorithm degenerates into an arithmetic of multiple random starting points; weak disturbance on the solution is difficult to promote the search out of local optimum. The paper applies the following method: setting $\lambda$ as a random integer in the interval $\left(0,\left\|s_{i}\right\|_{1} / 2\right)$, optionally chooses locations decoding $\lambda$ as 1 in the solution $s_{i}$ which would be replaced by the corresponding position value in current optimal solution, thus completes the disturbance. In summary, this paper designs a discrete artificial bee colony reconstruction (DABCR) with disturbance mechanism to solve the problem of multi-target localization, its specific process is as follows:

Step 1 (algorithms initialization) Initialize each parameter of algorithm. To initialize $S N$ food sources, and make the iteration number $t=0$.

Step 2. Calculate fitness function, find the cumulative probability of each solution for observation of bee selection .

Step 3. (employed bee phase) Use formula (8) with redefined subtraction and addition for neighborhood search in each solution.

Step 4. If a better solution is found, the current solution will be replaced, simultaneously setting searching count to zero, otherwise using Perturb $\left(s_{i}\right)$ function with probability $P_{\text {perturb }}$ for disturbing operation; if the resulting solution is better than the current one, then replace it, otherwise 1 will be incremented to the search count.

Step 5. (onlooker bee phase) Onlooker bees use cumulative probability to evaluate the selected solutions and chooses food source for neighborhood search in accordance with formula (9).

Step 6. To preserve the historic optimal solution.

Step 7. (scout bee phase) to analyze the searching accounts of all solutions; if the corresponding searching times of a solution are larger than the specified upper limit $C_{\max }$, then re-initialize a solution to replace it.

Step 8. making $t=t+1$, to determine whether the maximum number of iterations reaches $I_{\max }$, and if so, then output the optimal solution, the algorithm finishes; otherwise go to step 2 and begin the next iteration. 


\section{Equations and mathematics}

The simulation runs on the Matlab2012b. The monitored zone is a $20 \mathrm{~m} \times 20 \mathrm{~m}$ square area, the area is divided into 400 squares with side length of $1 \mathrm{~m}$; the parameter of measurement matrix is generated as: $P_{0}=-40 \mathrm{~dB}, N_{0}=2.5$, $D_{0}=1$. Parameters used in DABCR algorithm are as follows: the sum of employment and scout bees $\mathrm{SN}$ is 100 , and each takes up half. Maximum cycle count times $C_{\max }=30$, the disturbance probability $P_{\text {perturb }}=0.1$, the maximum number of iterations $I_{\max }=300$. Figure 1 shows the use of 25 sensor nodes $(M=25)$, with six targets $(K=$ $6)$, in the ideal environment with no interference, the target position can be accurately reconstructed. When $\mathrm{SNR}=25 \mathrm{~dB}$ and $10 \mathrm{~dB}$, the localization result gradually deteriorates along with the increase of noise.

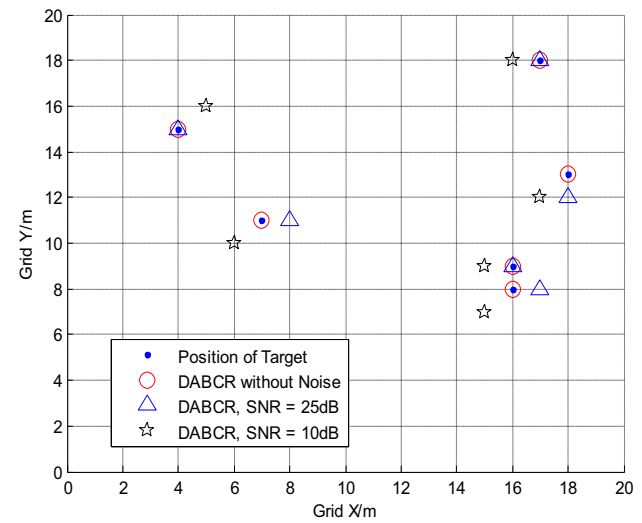

Figure 1. Location result of DABCR, with 6 targets

To test the error performance of node localization of different algorithms in different noise levels, the following parameter settings are used for the construction of test scenarios: $M=40, K=10$, SNR gradually reduces from $35 \mathrm{~dB}$ to $10 \mathrm{~dB}$. When SNR is less than $10 \mathrm{~dB}$, false targets begin to appear in the reconstructing results, the algorithm fails. In each SNR level 500 independent Monte Carlo experiments were carried out. Figure 2 shows the average value of the various SNR. As can be seen from Figure 2, RMSE changes more when SNR $<15 \mathrm{~dB}$, increasing with a larger margin. But in various noise levels the error of DABCR algorithm is still less than that of GMP and MLE algorithms.

Figure 3 shows a comparison of the performance of three algorithms with different objective numbers when $M=100$.

As can be seen from the Figure 3, when the target number is less than 20, three algorithms get more satisfactory localization effect in the absence of noise and SNR $=20 \mathrm{~dB}$; when the target number is greater than 20 , the reducing magnitude of accuracy of GMP and MLE algorithms is greater than that of DABCR algorithm. Meanwhile DABCR exhibits better robustness than GMP and MLE.

\section{Conclusion}

This paper presents a signal recovery method of compressed sensing based on discrete artificial bee colony algorithm for multi targeting in wireless sensor networks, and proposes a new food source location updating approach on the basis of discrete coding mode, designs disturbing operators to be applied to improve local search efficiency and enhance the effect of the global search, and verifies the effectiveness of the algorithm with comparison of simulation experiments.

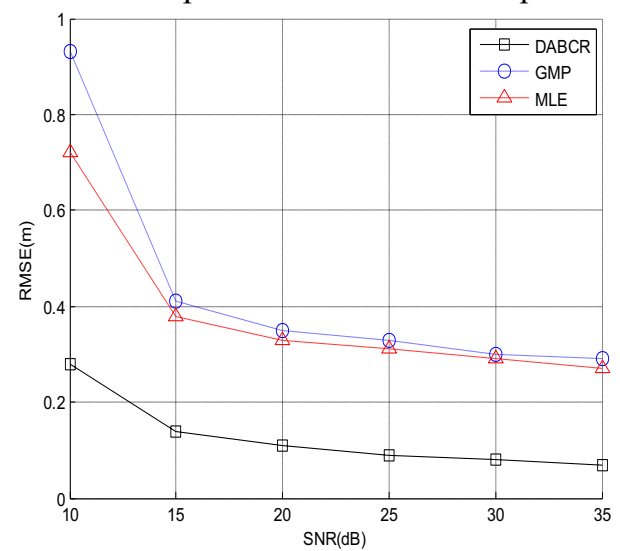

Figure 2. Comparison of RMSE under different SNRs

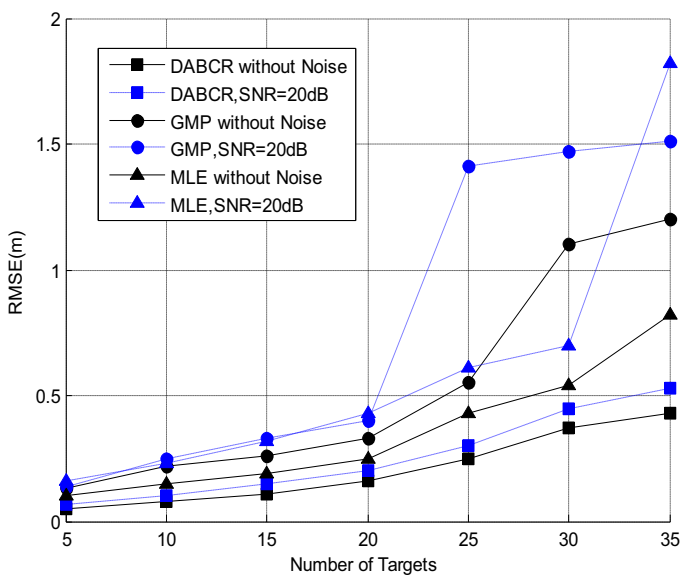

Figure 3. Comparison of RMSE for different target numbers and SNRs

\section{Acknowledgment}

This research work obtained the subsidisation of National Natural Science Foundation (Nos. 61563012, 61502290, 61363070); Guangxi Natural Science Foundation of China (No. 2014GXNSFAA118370); Guangxi Colleges and Universities Key Laboratory of cloud computing and complex systems (No. 15209). The authors would like to thank the (associate) editors and reviewers for their valuable and constructive comments and suggestions for improving this paper.

\section{References}

1. Akyildiz.I.F, Su.W, Sankarasubramaniam.Y, "Wireless sensor networks:A survey", Computer Networks, 38(2002).

2. Patwari.N et al., "Locating the nodes: cooperative localization in wireless sensor networks",IEEE Signal Processing Magazine, 22(2005). 
3. Chen.PC, "A nonline-of-sight error mitigation algorithm in location estimation" Proc. of the IEEE Wireless Communications Networking Conf., (1999).

4. Girod.L, Bychovskiy.v, Elson.J, Estrin.D, "Locating tiny sensors in time and space:A case study" Proc. of the 2002 IEEE International Conference on Computer Design: VLSI in Computers and Processors, (2002).

5. Girod.L, Estrin.D, "Robust range estimation using acoustic and multimodal sensing" Proc. of the IEEE/RSJ Int'1 Conf.on Intelligent Robots and Systems(IROS 2001).

6. Aksu.H, Aksoy.D, Kurpeoglu.I,"A study of localization metrics: Evaluation of position errors in wireless sensor networks", Computer Networks, 55, (2010).

7. Niculescu.D, Nath.B, "Ad hoc positioning system(APS) using AoA" Proc. IEEE INFOCOMM, (2003)

8. D. L. Donoho, "Compressive sensing ", IEEE Trans. Info. Theory, 52(2006).

9. Cevher.V, Duarte.M.F, Baraniuk.R.G, "Distributed target localization via spatial sparsity" Proc. of the European Signal Processing Conference, (2008).

10. F.Chen, S.Valaee, Z.Tan," Multiple target localization using compressive sensing" Proc. IEEE Global Communications Conference, (2009).

11. S.Nikitaki, P.Tsakalides, "Localization in wireless networks based on jointly compressed sensing" Proc. IEEE Signal Processing Conference,(2011).
12. Z.Bowu et al., "Sparse target counting and localization in sensor networks based on compressive sensing" Proc. INFOCOM, (2011).

13. W.Ju et al., "LCS: Compressive sensing based device-free localization for multiple targets in sensor networks" Proc. INFOCOM, (2013).

14. X.Qiang , Z.Wanting, "Adaptive Localization Algorithm Based on Distributed Compressed Sensing in Wireless Sensor Networks", Journal of Networks,9 (2014).

15. E.Candès, J.Romberg,T.Tao, "Robust uncertainty principles:exact signal reconstruction from highly incomplete frequency information", IEEE Transaction on Information Theory,.52, (2006).

16. E.Candès, J.Romberg, "Quantitative robust uncertainty principles and optimally sparse decompositions", Foundations of Compute Mathematics, 6 (2006).

17. E.Candès, M.B.Wakin, "An Introduction To Compressive Sampling",25 (2008).

18. D. Karaboga, B. Basturk, "A powerful and Efficient Algorithm for Numerical Function Optimization: Artificial Bee Colony (ABC) Algorithm", Journal of Global Optimization, 39(2007).

19. D. Karaboga, B. Akay, "A Comparative Study of Artificial Bee Colony Algorithm", Applied Mathematics and Computation, 214 (2009). 